\title{
Time Pressure During Primary Care Office Visits: a Prospective Evaluation of Data from the Healthy Work Place Study
}

\author{
Kriti Prasad, $B A^{1,2,3}$, Sara Poplau, BA ${ }^{1,2,3}$, Roger Brown, $P h D^{4}$, Steven Yale, $M D, M A C P^{5}$, \\ Ellie Grossman, MD, MPH', Anita B. Varkey, $M D^{7}$, Eric Williams, $P h D^{8}$, \\ Hannah Neprash, $P h D^{9}$, and Mark Linzer, MD, MACP ${ }^{1,2,3,10}$ for the Healthy Work Place \\ (HWP) Investigators
}

\begin{abstract}
'Division of General Internal Medicine, Hennepin Healthcare, Minneapolis, MN, USA; ${ }^{2}$ Office of Professional Worklife, Hennepin Healthcare, Minneapolis, MN, USA; ${ }^{3}$ Hennepin Healthcare Research Institute, Hennepin Healthcare, Minneapolis, MN, USA; ${ }^{4}$ School of Nursing, UW-Madison, Madison, WI, USA; ${ }^{5}$ University of Central Florida College of Medicine, Orlando, FL, USA; ${ }^{6}$ Cambridge Health Alliance, Cambridge, MA, USA; ${ }^{7}$ Loyola University Stritch School of Medicine, Maywood, IL, USA; ${ }^{8}$ University of Alabama, Tuscaloosa, AL, USA; ${ }^{9}$ University of Minnesota School of Public Health, Minneapolis, MN, USA; ${ }^{10}$ Department of Medicine, Hennepin Healthcare, Minneapolis, MN, USA.
\end{abstract}

BACKGROUND: The relationship between worklife factors, clinician outcomes, and time pressure during office visits is unclear.

OBJECTIVE: To quantify associations between time pressure, workplace characteristics, and clinician outcomes.

DESIGN: Prospective analysis of data from the Healthy Work Place randomized trial.

PARTICIPANTS: 168 physicians and advanced practice clinicians in 34 primary care practices in Upper Midwest and East Coast.

MAIN MEASURES AND METHODS: Time pressure was present when clinicians needed more time than allotted to provide quality care. Other metrics included work control, work pace (calm to chaotic), organizational culture and clinician satisfaction, stress, burnout, and intent to leave the practice. Hierarchical analysis assessed relationships between time pressure, organizational characteristics, and clinician outcomes. Adjusted differences between clinicians with and without time pressure were expressed as effect sizes (ESs).

KEY RESULTS: Sixty-seven percent of clinicians needed more time for new patients and 53\% needed additional time for follow-up appointments. Time pressure in new patient visits was more prevalent in general internists than in family physicians $(74 \%$ vs $55 \%, p<0.05)$, women versus men $(78 \%$ vs $55 \%, p<0.01)$, and clinicians with larger numbers of complex psychosocial (81\% vs 59\%, $p<$ $0.01)$ and Limited English Proficiency patients (95\% vs $57 \%, p<0.001)$. Time pressure in new patient visits was associated with lack of control, clinician stress, and intent to leave (ESs small to moderate, $p<0.05$ ). Time pressure in follow-up visits was associated with chaotic workplaces

Prior Presentations Presented at the annual meetings of the Society of General Internal Medicine, Denver, CO, April 2018

Electronic supplementary material The online version of this article (https://doi.org/10.1007/s11606-019-05343-6) contains supplementary material, which is available to authorized users.

Received November 30, 2018

Revised July 10, 2019

Accepted August 28, 2019

Published online December 3, 2019 and burnout (small to moderate ESs, $p$ 's <0.05). Time pressure improved over time in workplaces with values alignment and an emphasis on quality.

CONCLUSIONS: Time pressure, more common in women and general internists, was related to chaos, control and culture, and stress, burnout, and intent to leave. Future studies should evaluate these findings in larger and more geographically diverse samples.

KEY WORDS: time pressure; physician satisfaction; health services research; medical culture; primary care; workforce.

J Gen Intern Med 35(2):465-72

DOI: $10.1007 / \mathrm{s} 11606-019-05343-6$

(C) Society of General Internal Medicine 2019

\section{INTRODUCTION}

Physicians often feel pressed for time during the standard primary care office visit. In fact, studies have shown that physicians, in an effort to expedite patient visits, tend to redirect a patient's opening statement within $23.1 \mathrm{~s}$ and interrupt patients twice or more in $25 \%$ of visits. ${ }^{1,2}$ Recent studies have also affirmed that shorter visits not only are common but also can lead to unaddressed health care issues and a diminished depth of understanding of patient concerns. ${ }^{3,4}$ The Physicians Foundation survey of over 17,000 of America's physicians ${ }^{5}$ supported this view, as only $14 \%$ felt they had enough time to provide high-quality care. Many such concerning outcomes prompted examination of workplace conditions in primary care in the Minimizing Error, Maximizing Outcome (MEMO) Study, where 53\% of primary care providers reported experiencing time pressure. Work conditions in MEMO were strongly associated with adverse physician outcomes (stress, burnout, dissatisfaction, and intent to leave). ${ }^{6}$

Time pressure can be defined as the inability to complete necessary work in the time allotted. The Physician Worklife Study (PWS) showed that time pressure was related to satisfaction $^{7}$ and was higher in women. ${ }^{8}$ Recent studies in dentistry, ${ }^{9}$ 
information technology, ${ }^{10}$ and primary care ${ }^{11}$ confirm the adverse effects of time pressure in varied medical and non-medical settings. A sub-study from MEMO demonstrated the relationship between time pressure from electronic medical records (EMRs) and adverse clinician outcomes. ${ }^{12}$ A conceptual model (Fig. 1) based on work from PWS, MEMO, and the Healthy Work Place (HWP) study links time pressure with organizational, practice-based and personal variables, and clinician outcomes. This model formed the basis for this investigation.

The aim of this study is to explore relationships between time pressure, the work environment, and clinician outcomes. We hypothesized that there are clinician and organizational characteristics that relate to greater perceived time pressure. Specifically, we anticipated that work conditions-such as work control and work pace - and organizational culture characteristics - such as quality emphasis and values alignment - would be associated with time pressure. We also hypothesized that there are direct relationships between time pressure, work conditions, and clinician outcomes. Utilizing data from HWP, a longitudinal study of 34 primary care practices, we asked the following questions: How much more time than allotted do clinicians feel they need for new patient and follow-up appointments? Is time pressure associated with structural and cultural workplace characteristics? And is greater time pressure associated with adverse clinician outcomes?

\section{METHODS}

\section{Study Sample}

Our study is a prospective evaluation using data collected as part of the HWP study. HWP was a cluster randomized control trial in 34 clinical practices, with 168 clinicians (general internists, family physicians, and advanced practice clinicians) at 3 health systems in the Upper Midwest and in the East Coast. All eligible clinics at all three sites were enrolled. Recruitment for the study was carried out by site directors and research assistants at all clinics associated with each site either at team meetings or via email. Surveys were either mailed, given out by hand, or sent via email. Reminders were sent. Clinic sites were inner city, suburban, or rural, and many of the clinics were Federally Qualified Health Centers. Sitespecific Institutional Review Boards provided approval. For the current analyses, data were combined for clinicians in all 34 practices without separating them by intervention versus control site.

\section{Study Design}

Seventeen of the clinics underwent interventions to address clinician stress and burnout. These interventions, discussed in greater detail elsewhere, ${ }^{13}, 14$ included interventions in three categories: workflow redesign (e.g., pairing clinicians and medical assistants), improved communication between provider groups (e.g., monthly clinic meetings addressing patient care issues), and quality improvement programs in chronic disease management (thereby reducing clinician workload). Worklife perceptions and clinician outcomes were measured at baseline and results were reported only to the 17 intervention sites which then designed process improvement programs. After 12-18 months, all 34 clinics were reassessed.

The primary outcome for this study was perceived time pressure during new patient and follow-up visits, with time pressure defined as a clinician stating they needed more time than allotted for the particular type of visit. For the analyses, time pressure was evaluated as a binary outcome, that is, whether the clinician required more time than allotted or did not. Other worklife metrics included chaos (workplace atmosphere, graded from calm to chaotic on a 5-point scale), lack of work control (using a 14-item scale from the MEMO study, designed to measure control over office and patient care issues), ${ }^{6}$ values alignment with leaders (multiple items derived in MEMO and graded on a 4-point scale), and an emphasis on quality versus productivity (also from MEMO and graded on a

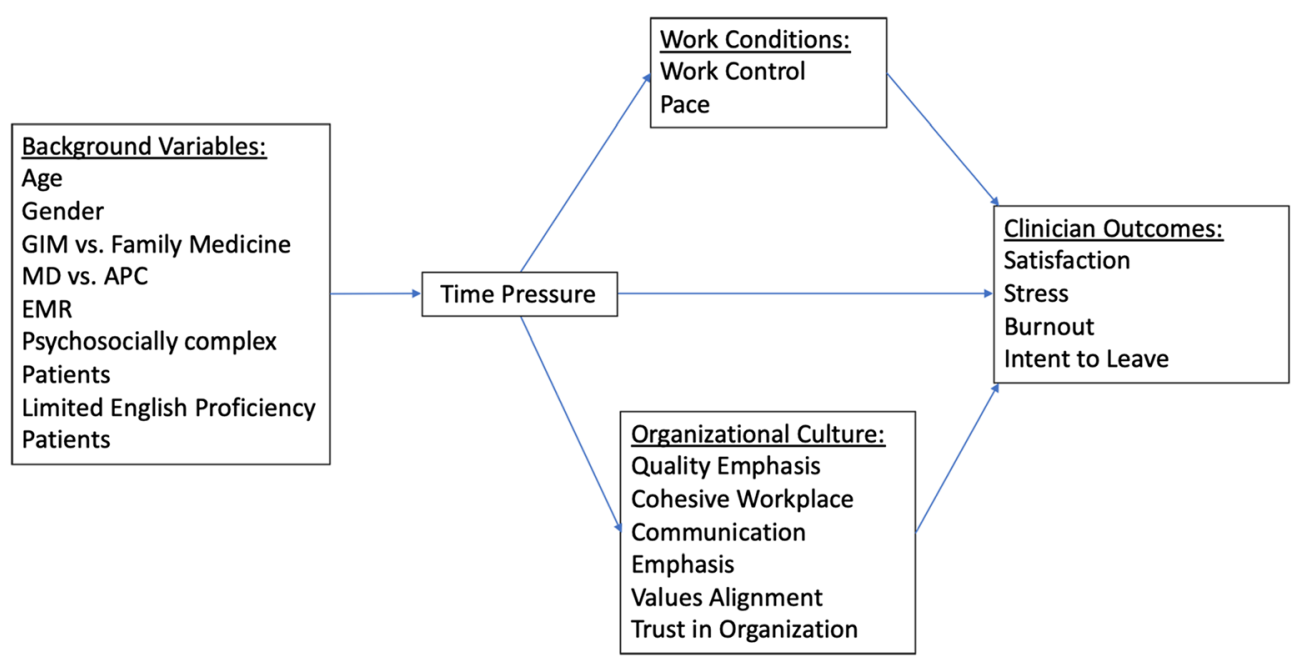

Figure 1 Conceptual model for interactions of work conditions and organizational characteristics with time pressure as they influence clinician outcomes. APC, advanced practice clinician; EMR, electronic medical record; GIM, general internal medicine; MD, medical doctor. 
4-point scale). Clinician outcomes included stress, satisfaction, burnout, and intent to leave the practice, which were measured on scales used in the Physician Worklife Study. ${ }^{7,15}$

\section{Analysis}

Univariate comparisons were calculated to assess the association of time pressure with personal characteristics, organizational characteristics, and clinician outcomes. Two-level multilevel mixed effects regression models, accounting for nesting of clinicians within clinics, were used to determine associations of the binary measure of time pressure with work conditions, organizational culture variables (e.g., values alignment, emphasis on quality), and clinician outcomes. Multilevel analysis allowed us to handle grouped data (clinicians within clinics), with observations being dependent, thus correctly modeling correlated error. These models then predicted marginal means for both work conditions, organizational culture, and clinician outcomes (stress, satisfaction, burnout, and intent to leave) controlling for clinician age, gender, practice type (family medicine vs general internal medicine), and physician versus nurse practitioner/physician assistant role. Models were performed at baseline and for time pressure improvement over time incorporating any treatment effects of the interventions. The two-level (clinic-clinician) linear random intercepts model is shown as:

$y_{i j}=\beta_{0} x_{0 i j}+\beta_{l} x_{I i j}+\sum_{h=1}^{H} \beta_{h, i j} x_{h, i j}+\mu_{0 j} x_{0 i j}+e_{0 i j} x_{0 i j}$

where $x_{1}=$ time pressure result for the $i$ th clinician in the $j$ th clinic, with $y_{i j}=$ the various clinician-related measures (e.g., perceived control, values alignment), with $x_{h, i j}$ clinician covariates (e.g., age, gender, specialty, and physician vs advanced practice clinician). The fixed portion of Eq. (1) includes the betas, with random portions of $u_{0 j} \sim N\left(0, \sigma 2_{v 0}\right)$ at the clinic level, and $e_{0 i j} \sim N\left(0, \sigma 2_{\varepsilon 0}\right)$ at the clinician level. Effect sizes were estimated as standardized parameters, with the effect size for continuous covariates obtained by standardizing both the covariate and the outcome measures, and with standardized parameters for the categorical covariates obtained by only standardizing the outcome measure. By convention, effect sizes were considered small at 0.2 , moderate at 0.5 , and large at 0.8 or higher. Analyses were performed using Stata v.15.1. ${ }^{16}$

Data from new and follow-up visits were analyzed separately. The binary measure of time pressure was assessed as whether a clinician stated they needed more time than allotted for new patient or follow-up visits. (Analyzing the data as a ratio of time needed to time allocated (that is, as a continuous variable showing greater or lesser time pressure) did not substantively alter the findings.) Improvements in time pressure were defined as binary, positive changes in time pressure from baseline to time 2, when clinics were reassessed 12 18 months later. A change in time pressure was considered an improvement if there was a reduction from experiencing time pressure at baseline to experiencing no time pressure at time 2 . Subjects were only included in this analysis if they provided full time pressure data at the two points in time (before and after the intervention clinics performed their interventions). Time was not a factor in the analyses, as we used difference in results between times $\mathrm{A}$ and $\mathrm{B}$ as the factor in the models. Once it was shown that time pressure was higher in females, separate regressions were run for female clinicians to determine key variables related to their experience of time pressure. All analyses were viewed as exploratory, in that we were willing to risk more alpha error so as not to miss identifying variables which may be associated with time pressure. In this regard, no adjustments were made for multiple comparisons.

\section{Role of Funding Source}

The Agency for Healthcare Research and Quality (AHRQ) provided funding for the study. There was no involvement of the funder in any aspect of study design, data analysis, interpretation, or writing of the manuscripts.

\section{RESULTS}

\section{Baseline Characteristics and Time Pressure}

The study included 168 clinicians, with 146 general internists and family physicians and 22 advanced practice clinicians (nurse practitioners and physician assistants) in 34 clinics in 3 separate geographic locations (Table 1). Of the clinicians, 52\% were female, and $64 \%$ were internists. Average time in practice was 12.8 years. Time pressure was higher, for the most part, in younger and female physicians, in physicians versus advanced practice clinicians, and in general internists versus family physicians. Time pressure was also seen more often in clinicians managing larger numbers of psychosocially complex and Limited English Proficiency (LEP) patients. There was no difference in percentage of clinicians with time pressure in control versus intervention groups at baseline (see Table 1). Likewise, there were no differences at time B (approximately 1 year after baseline measures), with $58 \%$ of 57 intervention clinicians with time pressure in new patient visits versus $53 \%$ of 64 controls $(p=0.598)$ and $49 \%$ of 57 intervention clinicians with time pressure in follow-up visits versus $43 \%$ of 65 controls $(p=0.503)$ (data not shown).

For new patient appointments (Table 2), the average time allotted was $35.2 \mathrm{~min}$ and the average time needed was $45.9 \mathrm{~min}(p<0.001)$. Sixty-seven percent of clinicians felt they needed more time than allotted for new patients (10.7 additional minutes, or $30 \%$ more time). For follow-up appointments, the average time allotted was $19.9 \mathrm{~min}$ and the average time needed was $23.5 \mathrm{~min}$ $(p<0.001)$, with $53 \%$ needing more time (average 3.6 additional minutes, or $18 \%$ more time needed). 
Table 1 Characteristics of Clinicians in the HWP Trial, by Time Pressure at Baseline

\begin{tabular}{|c|c|c|c|c|}
\hline & $N$ (time pressure/total) & Time pressure & Adequate time & $\overline{p \text { value }}$ \\
\hline \multicolumn{5}{|l|}{ Initial visit time pressure } \\
\hline Mean age/SD (years) & - & $46(8.86)$ & $49(9.54)$ & 0.070 \\
\hline \multicolumn{5}{|l|}{ Sex } \\
\hline Female & $65 / 83$ & $78 \%$ & $22 \%$ & \multirow[t]{2}{*}{0.002} \\
\hline Male & $42 / 77$ & $55 \%$ & $45 \%$ & \\
\hline \multicolumn{5}{|l|}{ Type of clinician } \\
\hline Physicians & $96 / 139$ & $69 \%$ & $31 \%$ & \multirow[b]{2}{*}{0.209} \\
\hline Advanced practice clinicians & $11 / 20$ & $55 \%$ & $45 \%$ & \\
\hline \multicolumn{5}{|l|}{ Specialty } \\
\hline General internal medicine & $75 / 101$ & $74 \%$ & $26 \%$ & \multirow[b]{2}{*}{0.013} \\
\hline Family medicine & $32 / 58$ & $55 \%$ & $45 \%$ & \\
\hline \multicolumn{5}{|l|}{ Race. } \\
\hline White & $87 / 132$ & $66 \%$ & $34 \%$ & \multirow[b]{2}{*}{0.272} \\
\hline Non-White & $20 / 26$ & $77 \%$ & $23 \%$ & \\
\hline \multicolumn{5}{|l|}{ Treatment groups (baseline) } \\
\hline Treatment & $54 / 78$ & $69 \%$ & $31 \%$ & \multirow[b]{2}{*}{0.648} \\
\hline Control & $52 / 79$ & $66 \%$ & $34 \%$ & \\
\hline \multicolumn{5}{|l|}{ Female patients } \\
\hline$<50 \%$ & $13 / 23$ & $57 \%$ & $43 \%$ & \multirow[b]{2}{*}{0.243} \\
\hline$\geq 50 \%$ & $93 / 135$ & $69 \%$ & $31 \%$ & \\
\hline \multicolumn{5}{|l|}{ Non-English-speaking patients } \\
\hline$<30 \%$ & $66 / 116$ & $57 \%$ & $43 \%$ & \multirow[b]{2}{*}{$<0.001$} \\
\hline$\geq 30 \%$ & $40 / 42$ & $95 \%$ & $5 \%$ & \\
\hline \multicolumn{5}{|c|}{ Patients with psychosocial problems } \\
\hline$<50 \%$ & $59 / 100$ & $59 \%$ & $41 \%$ & \multirow[b]{2}{*}{0.004} \\
\hline$\geq 50 \%$ & $47 / 58$ & $81 \%$ & $19 \%$ & \\
\hline \multicolumn{5}{|l|}{ Follow-up visit time pressure } \\
\hline Mean age/SD (years) & - & $46(9.14)$ & $48(9.03)$ & 0.122 \\
\hline Sex & & & & \multirow{3}{*}{$<0.05$} \\
\hline Female & $49 / 80$ & $61 \%$ & $39 \%$ & \\
\hline Male & $33 / 76$ & $43 \%$ & $57 \%$ & \\
\hline \multicolumn{5}{|l|}{ Type of clinician } \\
\hline Physicians & $77 / 137$ & $56 \%$ & $44 \%$ & \\
\hline Advanced practice clinicians & $5 / 19$ & $26 \%$ & $74 \%$ & 0.014 \\
\hline Specialty & & & & \\
\hline General internal medicine & $60 / 100$ & $60 \%$ & $40 \%$ & \\
\hline Family medicine & $22 / 56$ & $39 \%$ & $61 \%$ & 0.012 \\
\hline Race & & & & \\
\hline White & $63 / 129$ & $49 \%$ & $51 \%$ & \\
\hline Non-White & $18 / 26$ & $69 \%$ & $31 \%$ & 0.057 \\
\hline Treatment groups (baseline) & & & & \\
\hline Treatment & $38 / 76$ & $50 \%$ & $50 \%$ & \\
\hline Control & $43 / 78$ & $55 \%$ & $45 \%$ & 0.523 \\
\hline Female patients & & & & \\
\hline$<50 \%$ & $10 / 23$ & $43 \%$ & $57 \%$ & \\
\hline$\geq 50 \%$ & $72 / 132$ & $55 \%$ & $45 \%$ & 0.326 \\
\hline Non-English-speaking patients & & & & \\
\hline$<30 \%$ & $49 / 113$ & $43 \%$ & $57 \%$ & \\
\hline$\geq 30 \%$ & $33 / 52$ & $79 \%$ & $21 \%$ & $<0.001$ \\
\hline Patients with psycho-social pro & & & & \\
\hline$<50 \%$ & $42 / 98$ & $43 \%$ & $57 \%$ & \\
\hline$\geq 50 \%$ & $40 / 57$ & $70 \%$ & $30 \%$ & 0.001 \\
\hline
\end{tabular}

Unadjusted univariate biphasic comparisons

On average, women clinicians needed 14.0 additional minutes for new patient visits $(42 \%$ more time than allotted), while men required 7.4 additional minutes $(20 \%$ more time needed). For these new patient visits, $78 \%$ of women needed more time versus $55 \%$ of men $(p<0.01)$. For follow-up visits, women required 5.8 additional minutes $(30 \%$ more time than allotted) versus men requiring 2.8 additional minutes ( $15 \%$ more time), with $61 \%$ of women needing more time for follow-up visits versus $43 \%$ of men $(p<0.05)$. Regression analyses confirmed a gender effect, with higher time pressure found in women clinicians; key variables associated with time pressure in women clinicians were chaotic environments (pace of work) and large numbers of LEP patients.

\section{Time Pressure Relationships with Other Worklife Metrics}

In multilevel hierarchical models, time pressure during new patient appointments (Table 3) was significantly associated with work control (work control score of 2.28 for timepressured clinicians on a composite score of 14 items anchored from 1 to 4, vs 2.49 for those without time pressure, small to moderate $\mathrm{ES}-0.398, p=0.013)$. Organizational culture variables such as values alignment and quality emphasis appeared 
Table 2 Comparisons of Time Needed vs Time Allotted for New Patient and Follow-up Visits for All Clinicians, and for Male and Female Clinicians

\begin{tabular}{llll}
\hline \hline & Time allotted $($ min) & Time needed $($ min) & \% clinicians requiring more time than allotted \\
\hline New patient appointment & $35.2(\mathrm{SD}=10.4)$ & $45.9(\mathrm{SD}=10.5)^{* * * *}$ & 67 \\
Follow-up appointment & $19.9(\mathrm{SD}=3.5)$ & $23.5(\mathrm{SD}=6.0)^{* * *}$ & 53 \\
Male new patient & $36.7(\mathrm{SD}=10.8)$ & $44.1(\mathrm{SD}=11.1)^{* * * a}$ & $55^{\mathrm{b}}$ \\
Female new patient & $33.7(\mathrm{SD}=9.9)$ & $47.7(\mathrm{SD}=9.7)^{* * *}$ & 78 \\
Male follow-up & $19.1(\mathrm{SD}=3.4)$ & $21.9(\mathrm{SD}=5.3)^{* * \mathrm{a}}$ & $43^{\mathrm{b}}$ \\
Female follow-up & $19.2(\mathrm{SD}=3.7)$ & $25.0(\mathrm{SD}=6.3)^{* * *}$ & 61 \\
\hline
\end{tabular}

$* p<0.05 ; * * p<0.01 ; * * * p<0.001$. ${ }^{a}$ Comparison of males $v$ semales for number of minutes needed for a new patient $(44.1 \mathrm{vs} 47.7, p<0.05)$ and follow-up visit (21.9 vs 25.0, $p<0.001) .{ }^{b}$ Comparison of \% males vs females needing more time for a new patient (55\% vs $78 \%$, $\left.p=0.002\right)$ and followup visit $(43 \%$ vs $61 \%, p<0.05)$

not to be associated with new patient time pressure. However, time pressure for new patients was associated with significant differences in clinician outcomes including stress $(3.47$ in time-pressured individuals vs 3.15 on a 5-point scale in nontime-pressured clinicians, ES 0.417, $p=0.015$ ) and intent to leave the practice (2.14 on a 5-point scale vs 1.79 , ES 0.345 , $p=0.046$ ). Time pressure in follow-up visits (Table 4 ) showed significant associations with chaotic work environments (3.62 on a 5-point scale for those with time pressure vs 3.39 for those without, ES $0.335, p=0.040)$ and with burnout ( 2.51 on a 5point scale vs 2.15 , ES $0.384, p=0.017$ ).

Over the course of the study, perceived time pressure during new patient visits (Table 5) improved in 44 clinicians. The one organizational culture metric associated with improvement in time pressure for new patients was values alignment between clinicians and leaders (2.40 vs 2.11 on a 5 -point scale, ES $0.486, p=0.026$ ). Time pressure for follow-up visits (Table 6 ) improved in 37 clinicians who noted that their clinics had a higher emphasis on quality as opposed to productivity ( $2.90 \mathrm{vs}$ 2.66 on a 5-point scale, ES $0.428, p=0.030$ ) and greater values alignment between clinicians and leaders (2.31 vs 2.07 ES 0.411, $p=0.024$ ). Clinician outcomes (stress, satisfaction, burnout, and intent to leave) were for the most part more favorable with improved time pressure in new patient and follow-up visits, but the differences in scores between time-pressured and non-time-pressured clinicians were not statistically significant. Selected full two-level hierarchical models are included in the Appendix (Tables 7, 8, 9, and 10).

\section{DISCUSSION}

In this prospective evaluation of data from 168 clinicians in 34 medical practices, we found most clinicians felt they needed more time than allotted to care for their patients. This need was especially cogent for female clinicians and general internists. Time pressure was high in clinicians with larger numbers of psychosocially complex and Limited English Proficiency patients, more prevalent in chaotic workplaces where clinicians lacked work control, and associated with stress, burnout, and intent to leave the practice. Time pressure improved in settings with favorable aspects of organizational culture, namely high values alignment between clinicians and leaders and an emphasis on quality versus productivity.

Although time pressure in primary care is not new, 2, 4, 5, 7, ${ }^{17-22}$ it is troubling that despite numerous articles describing it, the prevalence of physicians experiencing time pressure continues to rise. Since the MEMO study in $2009,{ }^{6}$ time pressure

Table 3 Time Pressure for New Patient Visits Related to Work Conditions, Aspects of Organizational Culture, and Clinician Outcomes at Baseline

\begin{tabular}{|c|c|c|c|c|c|c|}
\hline \multirow{2}{*}{$\begin{array}{l}\text { Metrics } \\
\text { Work conditions }\end{array}$} & \multirow[t]{2}{*}{ Time pressure $(n=111)$} & \multirow[t]{2}{*}{ No time pressure $(n=52)$} & \multirow[t]{2}{*}{ Effect size $^{\mathrm{a}}$} & \multirow[t]{2}{*}{$p>|z|$} & \multicolumn{2}{|c|}{$\begin{array}{l}\text { Effect size } \\
95 \% \text { confidence } \\
\text { interval }\end{array}$} \\
\hline & & & & & & \\
\hline Work control & 2.28 & 2.49 & -0.398 & $0.013^{*}$ & -0.713 & -0.083 \\
\hline Work pace (chaos) & 3.56 & 3.37 & 0.272 & 0.107 & -0.059 & 0.604 \\
\hline \multicolumn{7}{|c|}{ Aspects of organizational culture } \\
\hline Quality emphasis & 2.85 & 2.85 & 0.000 & 0.997 & -.340 & 0.341 \\
\hline Cohesive workplace & 2.80 & 2.78 & 0.047 & 0.785 & -0.291 & 0.386 \\
\hline Communication emphasis & 3.20 & 3.15 & 0.119 & 0.493 & -0.221 & 0.459 \\
\hline Values alignment & 2.30 & 2.42 & -0.184 & 0.261 & -0.506 & 0.137 \\
\hline Trust in organization & 2.85 & 2.80 & 0.066 & 0.688 & -0.259 & 0.392 \\
\hline \multicolumn{7}{|l|}{ Clinician outcomes } \\
\hline Satisfaction & 3.72 & 3.84 & -0.177 & 0.292 & -0.507 & 0.152 \\
\hline Stress & 3.47 & 3.15 & 0.417 & $0.015 *$ & 0.079 & 0.755 \\
\hline Burnout & 2.39 & 2.22 & 0.192 & 0.270 & -0.149 & 0.534 \\
\hline Intent to leave & 2.14 & 1.79 & 0.345 & $0.046^{*}$ & 0.006 & 0.683 \\
\hline
\end{tabular}

${ }^{*} p<0.05 .{ }^{a}$ Standardized marginal mean difference 
Table 4 Time Pressure for Follow-up Patient Visit Related to Work Conditions, Aspects of Organizational Culture, and Clinician Outcomes at Baseline

\begin{tabular}{|c|c|c|c|c|c|c|}
\hline \multirow{2}{*}{$\begin{array}{l}\text { Metrics } \\
\text { Work conditions }\end{array}$} & \multirow[t]{2}{*}{ Time pressure $(n=85)$} & \multirow[t]{2}{*}{ No time pressure $(n=75)$} & \multirow[t]{2}{*}{ Effect size $^{\mathrm{a}}$} & \multirow[t]{2}{*}{$p>|z|$} & \multicolumn{2}{|c|}{$\begin{array}{l}\text { Effect size } \\
95 \% \text { confidence } \\
\text { interval }\end{array}$} \\
\hline & & & & & & \\
\hline Work control & 2.28 & 2.44 & -0.306 & $0.038^{*}$ & -0.596 & -0.017 \\
\hline Work pace (chaos) & 3.62 & 3.39 & 0.335 & $0.040^{*}$ & 0.015 & 0.655 \\
\hline \multicolumn{7}{|c|}{ Aspects of organizational culture } \\
\hline Quality emphasis & 2.80 & 2.89 & -0.164 & 0.313 & -0.483 & 0.154 \\
\hline Cohesive workplace & 2.78 & 2.82 & -0.073 & 0.646 & -0.384 & 0.238 \\
\hline Communication emphasis & 3.16 & 3.21 & -0.098 & 0.545 & -0.416 & 0.219 \\
\hline Values alignment & 2.26 & 2.42 & -0.266 & 0.073 & -0.557 & 0.024 \\
\hline Trust in organization & 2.81 & 2.86 & -0.091 & 0.550 & -0.391 & 0.208 \\
\hline \multicolumn{7}{|l|}{ Clinician outcome } \\
\hline Satisfaction & 3.71 & 3.82 & -0.157 & 0.316 & -0.464 & 0.150 \\
\hline Stress & 3.48 & 3.25 & 0.299 & 0.071 & -0.025 & 0.624 \\
\hline Burnout & 2.51 & 2.15 & 0.384 & $0.017 *$ & 0.070 & 0.698 \\
\hline Intent to leave & 2.18 & 1.87 & 0.307 & 0.061 & -0.014 & .629 \\
\hline
\end{tabular}

${ }^{*} p<0.05 .{ }^{a}$ Standardized marginal mean difference

prevalence for new patients has increased from 53 to $67 \%$ of HWP clinicians in $2015(p=0.002)$. Thus the primary care workplace, described as feeling like a "hamster on a wheel," 23 has continued to generate time pressure, which makes even more clinicians feel unable to complete their work. Health information technology and EMRs have been cited as additional sources of stress. Involving more than half of the time of the clinician workday, ${ }^{24}$ they have been associated with time pressure. ${ }^{12}$ Moreover, physicians experiencing time pressures due to excessive documentation outside of work have twice the odds of burnout compared with other physicians. ${ }^{25}$ These outcomes may be associated with concerns about the attractiveness of primary care to trainees. ${ }^{26,27}$

Early work from the Physician Worklife Study ${ }^{7}$ showed an association between time pressure and job dissatisfaction. Time pressure correlated negatively with 7 facets of job satisfaction, including autonomy, personal time, and patient care issues. Since then, numerous authors have shown the deleterious consequences of pressured primary care visits; ${ }^{4}$, ${ }^{17,}{ }^{19}$ HWP now extends those findings.

Our data show associations between time pressure, stress, burnout, and intent to leave. These outcomes are particularly important, as burnout is linked to adverse clinician outcomes (depersonalization and turnover) and patient outcomes (satisfaction, adherence, and quality), while intent to leave is linked to turnover and loss of practice income $(\$ 250,000-\$ 500,000$ per provider). ${ }^{28,29}$ Addressing predictors of stress (chaos, lack of control, and time pressure) would be reasonable targets for preventing burnout and improving practice sustainability.

Time pressure was more common in women clinicians, with women requiring $40 \%$ more time for new patient visits and men needing $20 \%$ more time. These gender differences may stem from differing expectations and case mix of patients seeing women versus men physicians. ${ }^{17}$ Women, for whom there are high expectations for listening, spend $10 \%$ more time than men for comparable visits; ${ }^{30}$ however, HWP data show

Table 5 Improvements in Time Pressure (New Patient Visits) Related to Work Conditions, Aspects of Organizational Culture, and Clinician Outcomes

\begin{tabular}{|c|c|c|c|c|c|c|}
\hline \multirow{2}{*}{$\begin{array}{l}\text { Metrics } \\
\text { Work conditions }\end{array}$} & \multirow[t]{2}{*}{$\begin{array}{l}\text { Estimated means } \\
\text { Improvement }(n=44)\end{array}$} & \multirow[t]{2}{*}{$\begin{array}{l}\text { Estimated means } \\
\text { No improvement }{ }^{\mathrm{a}}(n=75)\end{array}$} & \multirow[t]{2}{*}{ Effect size $^{b}$} & \multirow[t]{2}{*}{$p>|z|$} & \multicolumn{2}{|c|}{$\begin{array}{l}\text { Effect size } \\
95 \% \text { confidence } \\
\text { interval }\end{array}$} \\
\hline & & & & & & \\
\hline Work control & 2.14 & 2.21 & -0.160 & 0.423 & -0.553 & 0.232 \\
\hline Work pace (chaos) & 3.51 & 3.67 & -0.243 & 0.329 & -0.731 & 0.245 \\
\hline \multicolumn{7}{|c|}{ Aspects of organizational culture } \\
\hline Quality emphasis & 2.94 & 2.71 & 0.392 & 0.096 & -0.069 & 0.853 \\
\hline Cohesive workplace & 2.97 & 2.82 & 0.260 & 0.264 & -0.196 & 0.716 \\
\hline Communication emphasis & 3.16 & 3.10 & 0.119 & 0.640 & -0.382 & 0.622 \\
\hline Values alignment & 2.40 & 2.11 & 0.486 & $0.026^{*}$ & 0.058 & 0.913 \\
\hline Trust in organization & 2.85 & 2.85 & -0.008 & 0.968 & -0.425 & 0.408 \\
\hline \multicolumn{7}{|l|}{ Clinician outcomes } \\
\hline Satisfaction & 3.92 & 3.64 & 0.451 & 0.063 & -0.024 & 0.926 \\
\hline Stress & 3.26 & 3.51 & -0.317 & 0.190 & -0.791 & 0.156 \\
\hline Burnout & 2.29 & 2.50 & -0.250 & 0.233 & -0.663 & 0.161 \\
\hline Intent to leave & 2.02 & 2.38 & -0.317 & 0.212 & -0.815 & 0.180 \\
\hline
\end{tabular}

${ }^{*} p<0.05 .{ }^{a}$ Of 75 clinicians with no improvement, 54 had no change and 21 experienced worse time pressure. ${ }^{b}$ Standardized marginal mean difference. Forty-six subjects not included due to missing time pressure data at either time A or B 
Table 6 Improvements in Time Pressure (Follow-up Patient Visits) Related to Work Conditions, Aspects of Organizational Culture, and Clinician Outcomes

\begin{tabular}{|c|c|c|c|c|c|c|}
\hline \multirow{2}{*}{$\begin{array}{l}\text { Metrics } \\
\text { Work conditions }\end{array}$} & \multirow[t]{2}{*}{$\begin{array}{l}\text { Estimated means } \\
\text { Improvement }(n=37)\end{array}$} & \multirow[t]{2}{*}{$\begin{array}{l}\text { Estimated mean } \\
\text { No improvement }{ }^{\mathrm{a}}(n=80)\end{array}$} & \multirow[t]{2}{*}{ Effect size $^{b}$} & \multirow[t]{2}{*}{$p>|z|$} & \multicolumn{2}{|c|}{$\begin{array}{l}\text { Effect size } \\
95 \% \text { confidence } \\
\text { interval }\end{array}$} \\
\hline & & & & & & \\
\hline Work control & 2.16 & 2.24 & -0.170 & 0.332 & -0.516 & 0.174 \\
\hline Work pace (chaos) & 3.52 & 3.66 & -0.202 & 0.339 & -0.619 & 0.213 \\
\hline \multicolumn{7}{|c|}{ Aspects of organizational culture } \\
\hline Quality emphasis & 2.90 & 2.66 & 0.428 & $0.030 *$ & 0.041 & 0.814 \\
\hline Cohesive workplace & 2.92 & 2.80 & 0.204 & 0.275 & -0.162 & 0.571 \\
\hline Communication emphasis & 3.13 & 3.09 & 0.091 & 0.668 & -0.327 & 0.510 \\
\hline Values alignment & 2.31 & 2.07 & 0.411 & $0.024 *$ & 0.055 & 0.767 \\
\hline Trust in organization & 2.93 & 2.76 & 0.273 & 0.116 & -0.067 & 0.615 \\
\hline \multicolumn{7}{|l|}{ Clinician outcomes } \\
\hline Satisfaction & 3.79 & 3.62 & 0.273 & 0.195 & -0.139 & 0.687 \\
\hline Stress & 3.32 & 3.52 & -0.251 & 0.252 & -0.681 & 0.178 \\
\hline Burnout & 2.32 & 2.47 & -0.177 & 0.371 & -0.564 & 0.210 \\
\hline Intent to leave & 2.39 & 2.31 & 0.067 & 0.751 & -0.349 & 0.484 \\
\hline
\end{tabular}

${ }^{*} p<0.05 .{ }^{a}$ Of 80 clinicians with no improvement, 53 had no change and 27 experienced worse time pressure. ${ }^{b}$ Standardized marginal mean difference. Forty-eight subjects not included due to missing time pressure data at either time A or B

that up to $40 \%$ more time may be needed by women for new patient visits, and $30 \%$ more time for follow-up appointments. Some organizations have attempted to reduce gender disparities for women by adjusting panel sizes by patient gender. ${ }^{31}$ Other next steps might include allowing clinicians and patients to choose appropriate time needed for their subsequent visits. Patients of women clinicians have been shown to have lower mortality and readmission rates as compared with those of men, ${ }^{32}$ but our data suggest these outcomes may come at a personal cost due to higher time pressures during patient encounters.

Our study also highlights relationships between time pressure and challenging work conditions, including less control of the workplace and more chaotic workplaces. Lack of control is a major factor in producing stress, ${ }^{18}$ and chaos is associated with stress and medical errors. ${ }^{33}$ The typical primary care practice may thus become the "perfect storm" of adverse work conditions - chaos, lack of control, and time pressure - which are linked to stress, burnout, and intent to leave. If confirmed in larger studies, our findings suggest that assessing these factors prospectively could allow a practice to better determine how close it is to being a healthy workplace.

Our data show less time pressure in supportive workplaces, with high values alignment and an emphasis on quality over productivity. Workplaces that aligned values between leadership and clinicians showed improved time pressure for new and follow-up patient visits. Aligning values may make clinicians more willing to endure time pressure. Alternatively, more supportive workplaces may provide more trained staff to reduce administrative burden. Transformational leadership can moderate the impact of time pressure on information technology workers. ${ }^{10}$ Thus, organizational leaders can advocate for ways to reduce time pressure to improve both clinician engagement and stress.

\section{Limitations}

Our study has several limitations. Measures were predominantly self-reported, there were only 3 geographically separate sites, and time pressure improved in a minority of the clinicians. The number of clinicians was relatively small, and many of the effect sizes were small to moderate. While we enrolled $100 \%$ of eligible clinics, we do not know participation rates of clinicians; thus, there may have been bias in terms of which types of clinicians (burned out vs not burned out) chose to enroll. While the measures have been well validated in multiple settings, we still propose these findings be viewed as exploratory and needing confirmation in larger and more geographically diverse studies.

\section{CONCLUSION}

In sum, most HWP clinicians felt they needed more time with their patients to provide quality care. Because our data show that time pressure is associated with workplace chaos and stress, and that quality emphasis and values alignment between clinicians and leaders seem to diminish time pressure, time pressure may be an important and remediable metric in primary care.

Acknowledgments: HWP Investigators include those listed in the author group and Diane Kohnhorst, Marshfield Clinic, Marshfield WI; Jill Wallock, Loyola University School of Medicine, Chicago, IL; Michael Barbouche, Forward Health Group, Madison, WI; and Dr. Lanis Hicks, University of Missouri, Columbia, MO. Ms. Kohnhorst and Ms. Wallock were involved in data collection, Mr. Barbouche in study design, and Dr. Hicks in study design and data interpretation.

Corresponding Author: Mark Linzer, MD, MACP; Department of Medicine Hennepin Healthcare, 701 Park Ave (G5), Minneapolis, MN 55415, USA (e-mail: Mark.linzer@hcmed.org). 
Funding Information This study is supported the Agency for Healthcare Research and Quality (AHRQ) Grant No. 5R18HSO18160-03.

\section{Compliance with Ethical Standards:}

Site-specific Institutional Review Boards provided approval.

Conflict of Interest: Mark Linzer and Sara Poplau acknowledge support by the American Medical Association and the American College of Physicians for worklife training and oversight of ongoing projects. Dr. Linzer is supported by the Institute for Healthcare Improvement for his role in the Joy in Work initiative. All other authors declare that they have no conflicts of interest.

Healthy Workplace Investigators: Dr. Hicks receives royalties from a textbook; Mr. Barbouche is the Founder and CEO of a healthcare data management company in Madison, WI, Forward Health Group, and owns stock in the company.

\section{REFERENCES}

1. Marvel MK, Epstein RM, Flowers K, Beckman HB. Soliciting the Patient's Agenda: Have We Improved? JAMA. 1999;281(3):283-287. doi:https://doi.org/10.1001/jama.281.3.283

2. Rhoades DR, McFarland KF, Finch WH, Johnson AO. Speaking and Interruptions During Primary Care Office Visits. Fam Med.:5.

3. Geraghty EM, Franks P, Kravitz RL. Primary Care Visit Length, Quality and Satisfaction for Standardized Patients with Depression. J Gen Intern Med. 2007;22(12):1641-1647. doi:https://doi.org/10.1007/s11606007-0371-5

4. for the Association of Chiefs and Leaders in General Internal Medicine (ACLGIM) Writing Group*, Linzer M, Bitton A, et al. The End of the 15-20 Minute Primary Care Visit. J Gen Intern Med. 2015;30(11):1584-1586. https://doi.org/10.1007/s11606-015-3341-3

5. Hawkings M. 2016 Survey of America's Physicians. The Physicians Foundation; :84. https://physiciansfoundation.org/wp-content/uploads/2018/01/Biennial_Physician_Survey_2016.pdf.

6. Linzer M. Working Conditions in Primary Care: Physician Reactions and Care Quality. Ann Intern Med. 2009;151(1):28. doi:https://doi.org/10. 7326/0003-4819-151-1-200907070-00006

7. Linzer M, Konrad TR, Douglas J, et al. Managed Care, Time Pressure, and Physician Job Satisfaction: Results from the Physician Worklife Study. J Gen Intern Med. 2000;15(7):441-450. doi:https://doi.org/10. 1046/j.1525-1497.2000.05239.x

8. McMurray JE, Linzer M, Konrad TR, Douglas J, Shugerman R, Nelson $\mathbf{K}$. The Work Lives of Women Physicians. J Gen Intern Med. 2000;15(6):372-380. doi:https://doi.org/10.1111/j.1525-1497.2000. im9908009. $\mathrm{x}$

9. Plessas A, Nasser M, Hanoch Y, O'Brien T, Bernardes Delgado M, Moles D. Impact of time pressure on dentists' diagnostic performance. $J$ Dent. 2019;82:38-44. doi:https://doi.org/10.1016/j.jdent.2019.01.011

10. Syrek CJ, Apostel E, Antoni CH. Stress in highly demanding IT jobs: transformational leadership moderates the impact of time pressure on exhaustion and work-life balance. $J$ Occup Health Psychol. 2013;18(3):252-261. doi:https://doi.org/10.1037/a0033085

11. Tsiga E, Panagopoulou E, Sevdalis N, Montgomery A, Benos A. The influence of time pressure on adherence to guidelines in primary care: an experimental study. BMJ Open. 2013;3(4):e002700. doi:https://doi.org/ 10.1136/bmjopen-2013-002700

12. Babbott S, Manwell LB, Brown R, et al. Electronic medical records and physician stress in primary care: results from the MEMO Study. $J$ Am Med Inform Assoc. 2014;21(e1):e100-e106. doi:https://doi.org/10.1136/ amiajnl-2013-001875

13. Linzer M, Poplau S, Grossman E, et al. A Cluster Randomized Trial of Interventions to Improve Work Conditions and Clinician Burnout in Primary Care: Results from the Healthy Work Place (HWP) Study. J Gen Intern Med. 2015;30(8):1105-1111. doi:https://doi.org/10.1007/ s11606-015-3235-4
14. Linzer M, Sinsky CA, Poplau S, Brown R, Williams E, the Healthy Work Place Investigators. Joy In Medical Practice: Clinician Satisfaction In The Healthy Work Place Trial. Health Aff (Millwood). 2017;36(10):1808-1814. doi:https://doi.org/10.1377/hlthaff.2017.0790

15. Williams ES, Konrad TR, Linzer M, et al. Refining the measurement of physician job satisfaction: results from the Physician Worklife Survey. SGIM Career Satisfaction Study Group. Society of General Internal Medicine. Med Care. 1999;37(11):1140-1154.

16. StataCorp. Stata Statistical Software: Release 15. College Station, TX: StataCorp LLC; 2017.

17. Linzer M, Harwood E. Gendered Expectations: Do They Contribute to High Burnout Among Female Physicians? J Gen Intern Med. 2018;33(6):963-965. doi:https://doi.org/10.1007/s11606-018-4330-0

18. Linzer M, Gerrity M, Douglas JA, McMurray JE, Williams ES, Konrad TR. Physician stress: results from the physician worklife study. Stress Health. 2002;18(1):37-42. doi:https://doi.org/10.1002/smi.917

19. Warde C. Time Is of the Essence. J Gen Intern Med. 2001;16(10):712-713. doi:https://doi.org/10.1111/j.1525-1497.2001.08020.x

20. Linzer M, Levine R, Meltzer D, Poplau S, Warde C, West CP. 10 Bold Steps to Prevent Burnout in General Internal Medicine. J Gen Intern Med. 2014;29(1):18-20. doi:https://doi.org/10.1007/s11606-013-2597-8

21. Jaén CR, Stange KC, Nutting PA. Competing demands of primary care: a model for the delivery of clinical preventive services. J Fam Pract. 1994;38(2): 166-171.

22. Satterwhite $\mathbf{S}$, Knight KR, Miaskowski C, et al. Sources and Impact of Time Pressure on Opioid Management in the Safety-Net. J Am Board Fam Med. 2019;32(3):375-382. doi:https://doi.org/10.3122/jabfm.2019.03. 180306

23. Bitton A, Schwartz GR, Stewart EE, et al. Off the Hamster Wheel? Qualitative Evaluation of a Payment-Linked Patient-Centered Medical Home (PCMH) Pilot. Milbank Q. 2012;90(3):484-515. doi:https://doi.org/ $10.1111 / \mathrm{j} .1468-0009.2012 .00672 . \mathrm{x}$

24. Arndt BG, Beasley JW, Watkinson MD, et al. Tethered to the EHR: Primary Care Physician Workload Assessment Using EHR Event Log Data and Time-Motion Observations. Ann Fam Med. 2017;15(5):419-426. doi:https://doi.org/10.1370/afm.2121

25. Gardner RL, Cooper E, Haskell J, et al. Physician stress and burnout: the impact of health information technology. J Am Med Inform Assoc. 2019;26(2):106-114. doi:https://doi.org/10.1093/jamia/ocy145

26. Hauer KE, Durning SJ, Kernan WN, et al. Factors Associated With Medical Students' Career Choices Regarding Internal Medicine. JAMA. 2008;300(10):1154-1164. doi:https://doi.org/10.1001/jama.300.10. 1154

27. Dorsey ER, Jarjoura D, Rutecki GW. Influence of Controllable Lifestyle on Recent Trends in Specialty Choice by US Medical Students. JAMA. 2003;290(9): 1173-1178. doi:https://doi.org/10.1001/jama.290.9.1173

28. Buchbinder SB, Wilson M, Melick CF, Powe NR. Estimates of costs of primary care physician turnover. Am J Manag Care. 1999;5(11):14311438.

29. Schloss EP, Flanagan DM, Culler CL, Wright AL. Some Hidden Costs of Faculty Turnover in Clinical Departments in One Academic Medical Center. Acad Med. 2009;84(1):32. doi:https://doi.org/10.1097/ACM. Ob013e3181906dff

30. Roter DL, Hall JA, Aoki Y. Physician Gender Effects in Medical Communication: A Meta-analytic Review. JAMA. 2002;288(6):756. doi:https://doi.org/10.1001/jama.288.6.756

31. Trowbridge E, Bartels CM, Koslov S, Kamnetz S, Pandhi N. Development and Impact of a Novel Academic Primary Care Compensation Model. J Gen Intern Med. 2015;30(12):1865-1870. doi:https://doi.org/ $10.1007 / \mathrm{s} 11606-015-3410-7$

32. Tsugawa Y, Jena AB, Figueroa JF, Orav EJ, Blumenthal DM, Jha AK. Comparison of Hospital Mortality and Readmission Rates for Medicare Patients Treated by Male vs Female Physicians. JAMA Intern Med. 2017;177(2):206-213. doi:https://doi.org/10.1001/jamainternmed. 2016.7875

33. Perez HR, Beyrouty $\mathbf{M}$, Bennett $\mathbf{K}$, et al. Chaos in the Clinic: Characteristics and Consequences of Practices Perceived as Chaotic. $J$ Healthc Qual. 2017;39(1):43. doi:https://doi.org/10.1097/JHQ. 0000000000000016

Publisher's Note Springer Nature remains neutral with regard to jurisdictional claims in published maps and institutional affiliations. 